\title{
Association between lipid peroxidation and inflammation in obstructive sleep apnoea
}

\author{
K. Minoguchi*, T. Yokoe*, A. Tanaka*, S. Ohta*, T. Hirano*, G. Yoshino*, \\ C.P. O'Donnell ${ }^{\top}$ and M. Adachi*
}

ABSTRACT: In the present study, the authors examined the relationship between lipid peroxidation and inflammation in patients with obstructive sleep apnoea (OSA).

$A$ total of 40 obese patients with OSA were studied, along with 18 obese and 12 lean subjects without OSA. Overnight excretion of 8-isoprostane in urine and serum levels of high-sensitivity Creactive protein (hsCRP) were measured. In addition, the effects of 3 months' treatment with nasal continuous positive airway pressure (nCPAP) were studied in 20 obese patients with moderate-tosevere OSA.

Overnight urinary excretion of 8-isoprostane and serum levels of hsCRP were significantly higher in patients with moderate-to-severe OSA compared with patients with mild OSA and obese or lean subjects without OSA. Overnight urinary excretion of 8-isoprostane significantly correlated with apnoea-hypopnoea index, duration of hypoxia during sleep, body mass index, and serum levels of hsCRP in patients with OSA. The severity of OSA was an independent factor predicting the urinary excretion of 8-isoprostane. nCPAP significantly decreased urinary excretion of 8-isoprostane and serum levels of hsCRP.

In conclusion, these results suggest that both obstructive sleep apnoea severity and obesity can independently contribute to elevations in urinary excretion of 8-isoprostane. Therefore, obstructive sleep apnoea may increase the risks of cardiovascular morbidity in obese patients.

KEYWORDS: Cardiovascular diseases, C-reactive protein, hypoxia, inflammation, oxidative stress, sleep apnoea

O bstructive sleep apnoea (OSA) is associated with increased cardiovascular morbidity and mortality [1-3]. Although cardiovascular morbidity in patients who have OSA is not due to atherosclerosis alone, a recent study by MARTI et al. [4] showed that atherosclerosis may play an important role in cardiovascular mortality, as deaths from cardiovascular diseases in patients with untreated sleep apnoea are mainly due to acute myocardial infarction and stroke.

Ongoing inflammatory responses are involved in the pathogenesis of atherosclerosis [5, 6]. Recent epidemiological studies suggest that high-sensitivity (hs) C-reactive protein (CRP) is an important risk factor for atherosclerosis and coronary artery disease [7-9]. The present authors, along with others, have demonstrated that several inflammatory markers, including hsCRP, tumour necrosis factor- $\alpha$ and interleukin (IL)- 6 , are elevated in patients with OSA [10-12]. In addition, levels of circulating soluble adhesion molecules, such as intercellular adhesion molecule-1, vascular cell adhesion molecule- 1 and vascular endothelial growth factor, are elevated in patients with OSA [13, 14]. Moreover, serum levels of matrix metalloproteinase- 9 and carotid intimamedia thickness are also elevated in patients with OSA $[15,16]$. These findings suggest that OSA may increase the risks of atherosclerosis and cardiovascular diseases.

OSA is characterised by repetitive periods of upper airway collapse and results in cyclic periods of hypoxia/reoxygeneration that cause the increased generation of oxygen species by oxidative stress $[17,18]$. In fact, the production of oxygen species from neutrophils and monocytes obtained from patients with OSA was increased, and treatment with nasal continuous positive airway pressure (nCPAP) significantly decreased the production of oxygen species from these cells $[19,20]$. Moreover, oxidative damage is involved in the pathogenesis of atherosclerosis and cardiovascular diseases [21, 22]. Therefore, oxidative stress may contribute to the cardiovascular risk profile in patients with OSA.

In the cardiovascular system, lipids are in the first line of radical attack. 8-Isoprostane,
AFFILIATIONS

${ }^{*}$ First Dept of Internal Medicine,

Showa University,

"Division of Diabetes and

Endocrinology, Toho University,

Tokyo, Japan.

'Division of Pulmonary, Allergy, and Critical Care Medicine, University of Pittsburgh, Pittsburgh, PA, USA.

CORRESPONDENCE

K. Minoguchi

First Dept of Internal Medicine

Showa University

School of Medicine

1-5-8 Hatanodai

Shinagawa-ku

Tokyo 142-8666

Japan

Fax: 81337848742

E-mail: minochan@fn.catv.ne.jp

Received:

July 212005

Accepted after revision:

March 222006 
8-iso-prostaglandin $F 2_{\alpha}$, is one of the lipid markers for oxidative stress [23]. Due to its chemical stability, urinary excretion of 8-isoprostane is considered to be a reliable index of oxidant stress and ensuing lipid peroxidation in vivo. Although CARPAGNANO and co-workers [24, 25] demonstrated that 8isoprostane was increased in both peripheral blood and exhaled breath condensates from patients with OSA, overnight urinary excretion of 8-isoprostane was not studied in these patients. Moreover, the relationship between systemic lipid peroxidation (as evaluated by overnight urinary excretion of 8-isoprostane) and systemic inflammation (reflected by serum levels of hsCRP) has not been investigated in patients with OSA.

The purpose of this study was to extend the present authors' previous work examining the relationship between OSA and inflammation $[10,11,15,16]$. Specifically, the current authors determined the following in patients with OSA: 1) whether overnight urinary excretion of 8-isoprostane is elevated; 2) if overnight urinary excretion of 8-isoprostane is associated with increases in serum levels of hsCRP; 3) what factors are independent predictors for overnight urinary excretion of 8isoprostane; and 4) whether treatment with nCPAP decreases both overnight urinary excretion of 8 -isoprostane and serum levels of hsCRP.

\section{METHODS}

\section{Subjects}

A total of 40 males with abdominal obesity and newly diagnosed OSA, 18 snoring males with abdominal obesity but no OSA, and 12 lean, nonsnoring subjects without OSA were enrolled (table 1). Subjects with abdominal obesity and snoring, recruited from the outpatient clinic, were examined with polysomnography (PSG) and were classified based on their apnoea-hypopnoea index (AHI) as either OSA subjects or obese subjects without OSA. The 12 lean subjects were also examined with PSG and were classified as nonsnoring subjects without OSA. Lean and obese subjects without OSA were enrolled in order to investigate the independent effect of obesity on systemic lipid peroxidation and inflammation. Moreover, obese patients with OSA were separated into mild, moderate or severe OSA to investigate the effect of severity of OSA on systemic lipid peroxidation and inflammation. Before enrolment, all subjects were asked about their regular medications and medical history, including hypertension, cardiovascular diseases and cerebrovascular diseases, and were checked by chest radiograph, respiratory function, ECG, mouth-nose examination and systemic physical examination. Hypertension was defined by a casual blood pressure $\geqslant 140 / 90 \mathrm{mmHg}$ or by current use of antihypertensive agents. Diabetes mellitus was defined by a fasting blood glucose level $\geqslant 126 \mathrm{mg} \cdot \mathrm{dL}^{-1}$ or by use of glucose-lowering agents. Obesity was defined by a body mass index $(B M I) \geqslant 25$. All subjects who were free of the above and other diseases and were taking no medications were included in the present study. Subjects who smoked or had systemic infections at the time of the study, or within 2 weeks before the study, were also excluded. The study was approved by the ethics committee of Showa University (Tokyo, Japan), and all patients gave written informed consent.

\section{Polysomnography}

Full PSG monitoring was performed with the Compumedics P-series Sleep System (Compumedics Sleep, Abbotsford,
Australia). Electroencephalography (EEG), electrooculography (EOG), electromyography (EMG) and electrocardiography were performed simultaneously. Surface electrodes were used to record two channels of EEG (C3/A2, C4/A1), right and left EOGs, and submental EMG. Ventilatory flow at the nose and mouth was measured with thermistors. Ventilatory movements of the chest and abdomen were monitored by inductive plethysmography bands. The arterial oxygen saturation $\left(\mathrm{Sa}, \mathrm{O}_{2}\right)$ was measured transcutaneously with fingertip pulse oximetry. Apnoea was defined as continuous cessation of airflow for $>10 \mathrm{~s}$, and hypopnoea was defined as a reduction in airflow for $>10 \mathrm{~s}$ with an oxygen desaturation of $\geqslant 4 \%$ or an EEG arousal from sleep. Apnoeas were classified as obstructive, mixed or central according to standard criteria by the American Academy of Sleep Medicine [26]. The AHI was calculated as the total number of episodes of apnoea and hypopnoea per hour of sleep. An AHI of $\geqslant 5 \cdot h^{-1}$ in conjunction with sleep-related symptoms was considered diagnostic of OSA. An AHI of $\geqslant 5 \cdot h^{-1}$ and $<15 \cdot h^{-1}$ was classified as mild OSA, $\geqslant 15 \cdot h^{-1}$ and $<30 \cdot h^{-1}$ was classified as moderate OSA, and $\geqslant 30 \cdot \mathrm{h}^{-1}$ was classified as severe OSA. PSG was performed from 21:00-06:00 h. Of the 40 obese patients with OSA, 20 were considered to have mild OSA and 20 were considered to have moderate-to-severe OSA. The Epworth Sleepiness Scale (ESS) was used to investigate changes in subjective daytime sleepiness [27].

\section{Measurement of 8-isoprostane and hsCRP}

All subjects went to bed at 21:00 h and were woken at 06:00 h. Samples of peripheral venous blood were collected at 06:00 h after PSG was performed. In addition, urine was stored from 21:00-06:00 $\mathrm{h}$ and overnight urinary excretion of 8-isoprostane was measured with an enzyme immunoassay (Cayman Chemical Co., Ann Arbor, MI, USA) and corrected by urinary creatinine levels. Samples were stored at $-80^{\circ} \mathrm{C}$ until assay. hsCRP was measured with a latex particle-enhanced immunoturbidimetric assay as described previously [28].

\section{nCPAP treatment}

A total of 20 patients with moderate-to-severe OSA slept while using the automatic titration device (AutoSet Portable II Plus; ResMed, North Ryde, Australia). The following night, these patients slept with an autoCPAP machine using the AutoSet-S (ResMed). Afterwards, the patients were sent home. The duration of nCPAP treatment was 3 months. The mean daily duration of nCPAP use was $4.8 \pm 1.3 \mathrm{~h}$ in patients with moderate-to-severe OSA. Patients with moderate-to-severe OSA were admitted after 3 months of treatment and PSG was performed while patients received nCPAP. Urine was stored from 21:00-06:00 h and overnight urinary excretion of 8isoprostane was measured. Samples of venous blood were obtained at 06:00 h, and serum levels of hsCRP were measured again.

\section{Statistical analysis}

When comparing four groups, data were analysed by ANOVA with Bonferroni correction. Correlations were determined using Spearman's rank correlation coefficient. To assess the relative strength of association of overnight urinary excretion of 8-isoprostane with possible contributing factors, a stepwise multiple regression analysis was employed for patients with OSA. In the analysis, the present authors used overnight 


\begin{tabular}{|c|c|c|c|c|}
\hline Age yrs & $47.9 \pm 2.2$ & $48.1 \pm 2.4$ & $50.3 \pm 1.5$ & $49.3 \pm 3.5$ \\
\hline \multicolumn{5}{|l|}{ Blood pressure $\mathrm{mmHg}$} \\
\hline Systolic & $126.8 \pm 3.0$ & $130.1 \pm 3.3$ & $129.4 \pm 2.5$ & $132.0 \pm 3.9$ \\
\hline Waist cm & $74.3 \pm 3.1$ & $88.4 \pm 1.9^{\#}$ & $89.5 \pm 1.0^{\circ}$ & $90.5 \pm 1.8^{+}$ \\
\hline Waist/hip ratio & $0.78 \pm 0.01$ & $0.89 \pm 0.01^{\#}$ & $0.87 \pm 0.01^{\circ}$ & $0.92 \pm 0.02^{+}$ \\
\hline Total cholesterol $\mathrm{mg} \cdot \mathrm{dL}^{-1}$ & $200.6 \pm 7.4$ & $201.5 \pm 8.8$ & $202.0 \pm 6.2$ & $206.4 \pm 8.2$ \\
\hline $\mathrm{HDL}$ cholesterol $\mathrm{mg} \cdot \mathrm{dL}^{-1}$ & $45.5 \pm 3.3$ & $46.6 \pm 1.7$ & $41.4 \pm 1.4^{\bullet, \S}$ & $38.9 \pm 1.3^{+, f}$ \\
\hline LDL cholesterol $\mathrm{mg} \cdot \mathrm{dL}^{-1}$ & $120.0 \pm 5.5$ & $131.2 \pm 3.9$ & $135.0 \pm 2.5^{\circ}$ & $136.1 \pm 2.9^{+}$ \\
\hline$A H I$ events $\cdot h^{-1}$ & $3.4 \pm 0.5$ & $4.0 \pm 0.3$ & $9.8 \pm 2.0^{\oplus, 5}$ & $46.5 \pm 9.8^{+, f, \# \#}$ \\
\hline $\mathrm{Sa}, \mathrm{O}_{2}<90 \%$ \%TST & 0 & 0 & $4.1 \pm 3.1^{\bullet, 5}$ & $38.9 \pm 12.9^{+, f, \# \#}$ \\
\hline Lowest $\mathrm{Sa}, \mathrm{O}_{2} \%$ & $96.0 \pm 2.0$ & $96.4 \pm 1.8$ & $83.6 \pm 6.0^{\bullet, \S}$ & $70.9 \pm 7.9^{+, f, \# \#}$ \\
\hline Arousal index events $\cdot h^{-1}$ & $13.9 \pm 7.7$ & $15.4 \pm 9.8$ & $18.4 \pm 4.0^{\bullet . \S}$ & $42.3 \pm 6.8^{+, f, \# \#}$ \\
\hline TST min & $403.9 \pm 90.2$ & $411.9 \pm 100.1$ & $380.0 \pm 35.4^{\bullet, \S}$ & $328.0 \pm 68.1^{+, f, \# \#}$ \\
\hline ESS & $7.3 \pm 0.4$ & $8.2 \pm 0.5$ & $9.8 \pm 0.7^{\bullet}$ & $13.1 \pm 2.8^{+, f, \# \#}$ \\
\hline
\end{tabular}

Data are presented as mean \pm SEM, unless otherwise stated. BMI: body mass index; HDL: high-density lipoprotein; LDL: low-density lipoprotein; FVC: forced vital capacity; FEV1: forced expiratory volume in one second; \% pred: percentage of predicted; AHI: apnoea-hypopnoea index; Sa, $\mathrm{O}_{2}$ : arterial oxygen saturation; TST: total sleep time; ESS: Epworth Sleepiness Scale. ${ }^{\#}: p<0.01$ healthy control versus obese control; ${ }^{*}: p<0.01$ healthy control versus mild OSA; ${ }^{+}: p<0.01$ healthy control versus moderate-tosevere OSA; $;$ : $p<0.01$ obese control versus mild OSA; $;: p<0.01$ obese control versus moderate-to-severe OSA; ${ }^{\# \#: ~} p<0.01$ mild OSA versus moderate-to-severe OSA.

urinary excretion of 8-isoprostane as a dependent variable and evaluated the order of inclusion in the model of the following independent variables: age, BMI, ESS, waist/hip ratio, PSG variables, metabolic variables, and levels of hsCRP. Data are presented as mean \pm SEM, and a $p$-value $<0.05$ was considered statistically significant.

\section{RESULTS}

\section{Subject characteristics}

Characteristics of the 12 lean and 18 obese subjects without OSA, 20 obese patients with mild OSA, and 20 obese patients with moderate-to-severe OSA, including age, BMI, blood pressure, metabolic variables, PSG variables, respiratory function and ESS, are shown in table 1. AHI, percentage of time with $\mathrm{Sa}_{1} \mathrm{O}_{2}<90 \%$, arousal index and ESS were significantly higher in patients with moderate-to-severe OSA than in lean subjects without OSA, obese subjects without OSA, or patients with mild OSA. Lowest $\mathrm{Sa}_{1} \mathrm{O}_{2}$ and total sleep time were significantly lower in patients with moderate-to-severe OSA than in lean subjects without OSA, obese subjects without OSA or patients with mild OSA. Moreover, presence of snoring and OSA did not alter respiratory function.

\section{Overnight urinary excretion of 8-isoprostane and serum levels of hsCRP}

Overnight urinary excretion of 8-isoprostane in patients with

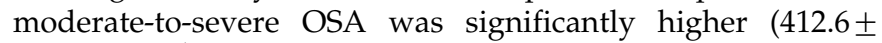
$\left.50.5 \mathrm{pg} \cdot \mathrm{mL}^{-1}\right)$ than in lean subjects without OSA $(111.3 \pm$ $7.9 \mathrm{pg} \cdot \mathrm{mL}^{-1} ; \mathrm{p}<0.0001$; fig. $\left.1 \mathrm{a}\right)$, obese subjects without OSA $\left(211.9 \pm 11.6 \mathrm{pg} \cdot \mathrm{mL}^{-1} ; \mathrm{p}<0.001\right)$ or patients with mild OSA $\left(257.4 \pm 16.7 \mathrm{pg} \cdot \mathrm{mL}^{-1} ; \mathrm{p}<0.01\right.$; fig. 1a). In addition, serum levels of hsCRP in patients with moderate-to-severe OSA $\left(0.23 \pm 0.05 \mathrm{mg} \cdot \mathrm{dL}^{-1}\right)$ were significantly higher than in lean subjects without OSA $\left(0.05 \pm 0.001 \mathrm{mg} \cdot \mathrm{dL}^{-1} ; \mathrm{p}<0.01\right)$, obese subjects without OSA $\left(0.07 \pm 0.03 \mathrm{mg} \cdot \mathrm{dL}^{-1} ; \mathrm{p}<0.01\right)$ or patients with mild OSA $\left(0.09 \pm 0.02 \mathrm{mg} \cdot \mathrm{dL}^{-1} ; \mathrm{p}<0.01\right.$; fig. $\left.1 \mathrm{~b}\right)$.

\section{Correlations between overnight urinary excretion of 8- isoprostane and different variables in patients with OSA}

Overnight urinary excretion of 8-isoprostane was positively correlated with AHI $(\mathrm{r}=0.55 ; \mathrm{p}=0.0004$; fig. $2 \mathrm{a})$, percentage of time with $\mathrm{Sa}_{1} \mathrm{O}_{2}<90 \%(\mathrm{r}=0.40 ; \mathrm{p}=0.02$; fig. $2 \mathrm{~b})$, serum levels of hsCRP ( $r=0.50 ; p=0.002$; fig. $2 \mathrm{c}$ ) and BMI ( $r=0.40 ; p=0.02$; fig. 2d) but was not significantly correlated with age, ESS or metabolic variables (table 2). Thus, in males with OSA, an increase in overnight urinary excretion of 8-isoprostane was related to the severity of OSA with longer duration of hypoxaemia and to higher serum levels of hsCRP and BMI.

Stepwise multiple regression analysis in patients with OSA To examine independent predictors of overnight urinary excretion of 8-isoprostane in patients with OSA, a stepwise multiple regression analysis was performed. After adjusting for age, ESS, PSG variables and metabolic variables, including BMI and triglyceride levels, AHI $(p=0.02)$ was the only significant independent predictor of overnight urinary excretion of 8-isoprostane (table 3). 

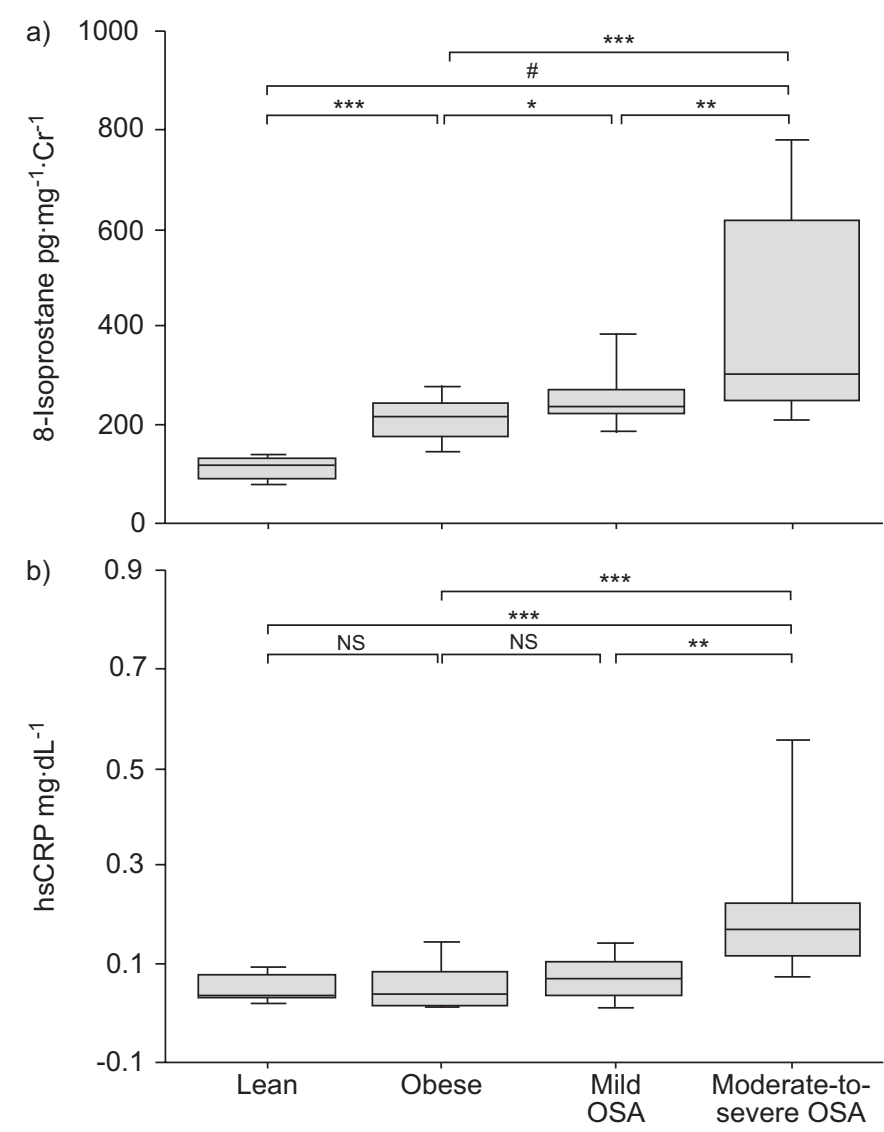

FIGURE 1. a) Overnight urinary excretion of 8-isoprostane and b) serum levels of high-sensitivity $\mathrm{C}$-reactive protein (hsCRP) in lean subjects without obstructive sleep apnoea (OSA; $n=12)$, obese subjects without OSA $(n=18)$, obese patients with mild OSA $(n=20)$ and obese patients with moderate-to-severe OSA $(n=20)$. The boundaries of each box represents the interquartile range (25th-75th centiles), the horizontal line inside the box represents the median, and the error bars outside the box represent the $95 \%$ confidence intervals of the mean. ${ }^{*}: p<0.05$; ${ }^{*}$ : $p<0.01$; ***: $p<0.001 ;{ }^{*}: p<0.0001$; NS: nonsignificant.

\section{Effect of nCPAP on overnight urinary excretion of 8- isoprostane and serum levels of hsCRP in patients with moderate-to-severe OSA}

In 20 patients with moderate-to-severe OSA who successfully completed 3 months of treatment, BMI did not change significantly and no new cardiovascular diseases were detected during the 3-month treatment with nCPAP. Treatment with nCPAP significantly decreased the AHI $\left(46.5 \pm 9.8\right.$ to $2.8 \pm 0.9$ events $\left.\cdot \mathrm{h}^{-1} ; \mathrm{p}<0.001\right)$, increased the lowest nocturnal $\mathrm{Sa}_{1} \mathrm{O}_{2}(70.9 \pm 7.9$ to $92.0 \pm 3.9 \% ; \mathrm{p}<0.001)$ and total sleep time $(328.0 \pm 68.1$ to $418.3 \pm 49.6 \mathrm{~min} ; \mathrm{p}<0.01)$, and decreased the percentage of time with $\mathrm{Sa}_{\mathrm{a}} \mathrm{O}_{2}<90 \%(38.9 \pm 12.9$ to $0.12 \pm 0.3 \% ; \mathrm{p}<0.001)$ and the arousal index $(42.3 \pm 6.8$ to $\left.16.2 \pm 3.4 \cdot h^{-1} ; \mathrm{p}<0.01\right)$. Treatment with $\mathrm{nCPAP}$ also significantly decreased overnight urinary excretion of 8-isoprostane $\left(412.6 \pm 50.5\right.$ to $246.2 \pm 11.9 \mathrm{pg} \cdot \mathrm{mL}^{-1} ; \mathrm{p}<0.03$; fig. 3a). In addition, serum levels of hsCRP were significantly decreased by the treatment with nCPAP $\left(0.23 \pm 0.05\right.$ to $0.11 \pm 0.02 \mathrm{pg} \cdot \mathrm{mL}^{-1}$; $\mathrm{p}<0.03$; fig. 3b).

\begin{tabular}{|c|c|c|}
\hline TABLE 2 & $\begin{array}{l}\text { Correlation coefficients bet } \\
\text { urinary excretion of } 8 \text {-isopro } \\
\text { variables in patients with ok } \\
\text { apnoea }\end{array}$ & $\begin{array}{l}\text { ernight } \\
\text { and different } \\
\text { e sleep }\end{array}$ \\
\hline Variables & 8-Isoprostane & p-value \\
\hline Age & 0.20 & NS \\
\hline AHI & 0.55 & 0.0004 \\
\hline $\mathrm{Sa}, \mathrm{O}_{2}<90 \%$ & 0.40 & 0.02 \\
\hline Cholesterol & 0.11 & NS \\
\hline Triglyceride & 0.01 & NS \\
\hline ESS & 0.18 & NS \\
\hline BMI & 0.40 & 0.02 \\
\hline Waist & 0.29 & NS \\
\hline Waist/hip ratio & 0.10 & NS \\
\hline hsCRP & 0.50 & 0.002 \\
\hline
\end{tabular}

Changes in AHI after 3 months' treatment with nCPAP were correlated with changes in overnight urinary excretion of 8isoprostane $(r=0.72 ; p=0.01)$ and serum levels of hsCRP $(\mathrm{r}=0.62 ; \mathrm{p}=0.01)$. In addition, changes in the percentage of time with $\mathrm{Sa}_{1} \mathrm{O}_{2}<90 \%$ after 3 months' treatment with nCPAP were correlated with changes in overnight urinary excretion of 8-isoprostane $(r=0.63 ; p=0.03)$ and serum levels of hsCRP $(\mathrm{r}=0.51 ; \mathrm{p}=0.04)$. Moreover, changes in overnight urinary excretion of 8-isoprostane after treatment with nCPAP correlated with changes in serum levels of hsCRP $(r=0.68 ; p=0.02)$ in patients with moderate-to-severe OSA.

\section{DISCUSSION}

In the present study, it was found that overnight urinary excretion of 8-isoprostane and serum levels of hsCRP in patients with moderate-to-severe OSA were significantly

\begin{tabular}{|c|c|c|c|}
\hline TABLE 3 & $\begin{array}{l}\text { lultiple regressio } \\
\text {-isoprostane leve }\end{array}$ & alysis & \\
\hline Variables & $\begin{array}{c}\text { Partial regression } \\
\text { coefficient }(\boldsymbol{\beta})\end{array}$ & SEM & p-value \\
\hline AHI & 0.68 & 0.27 & 0.02 \\
\hline $\mathrm{Sa}, \mathrm{O}_{2}<\mathbf{9 0 \%}$ & -0.25 & 0.27 & 0.35 \\
\hline hsCRP & 0.27 & 0.14 & 0.07 \\
\hline Age & -0.01 & 0.12 & 0.94 \\
\hline Triglyceride & -0.08 & 0.12 & 0.50 \\
\hline Total cholesterol & 0.09 & 0.14 & 0.55 \\
\hline BMI & 0.15 & 0.13 & 0.27 \\
\hline Waist/hip ratio & -0.04 & 0.13 & 0.74 \\
\hline ESS & -0.08 & 0.14 & 0.58 \\
\hline
\end{tabular}

AHI: apnoea-hypopnoea index; $\mathrm{Sa}_{1} \mathrm{O}_{2}$ : arterial oxygen saturation; hsCRP: highsensitivity C-reactive protein; BMI: body mass index; ESS: Epworth Sleepiness Scale. Coefficient of determination: $r^{2}=0.35 ; p<0.01$. 

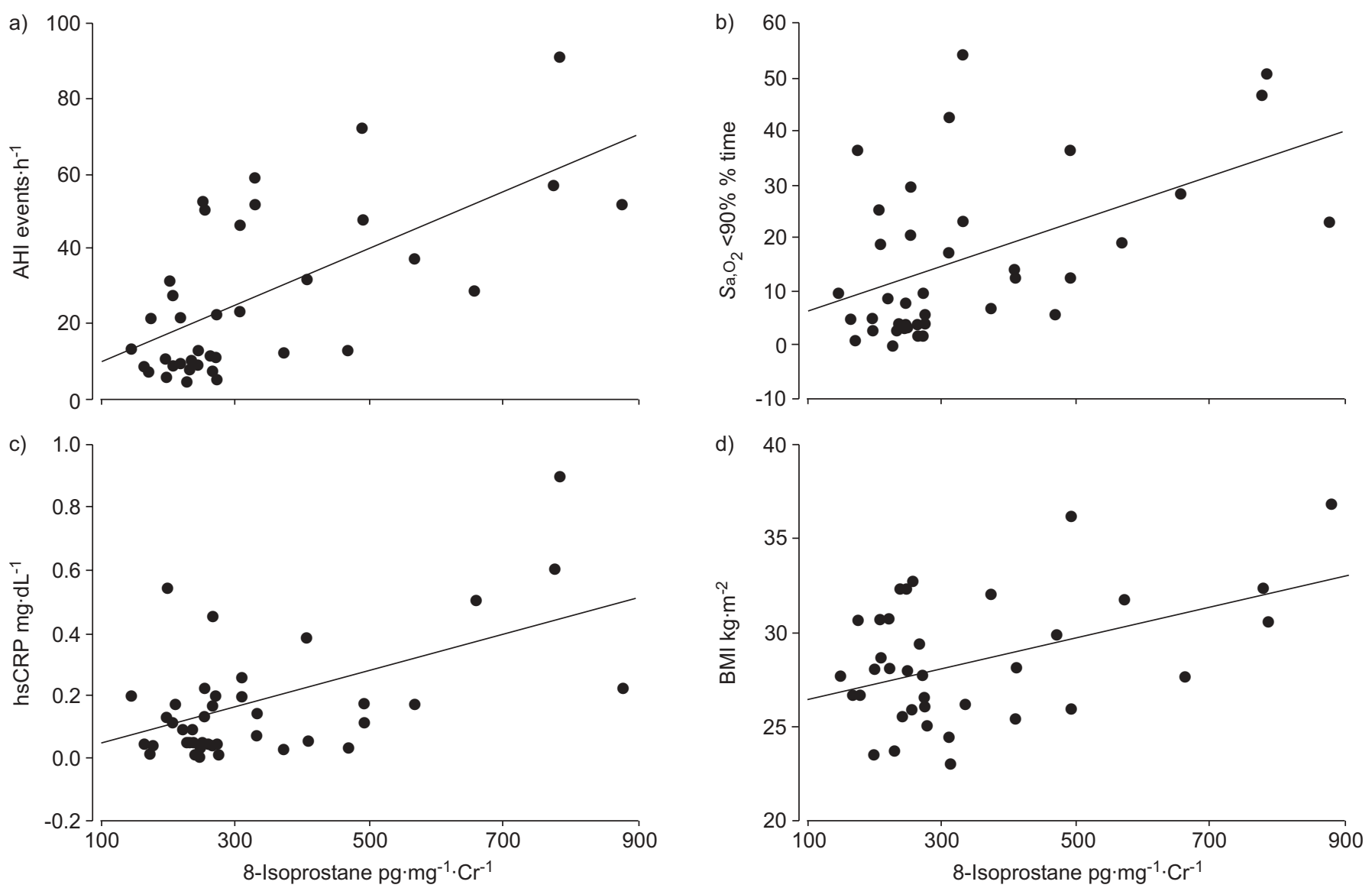

FIGURE 2. Correlations between overnight urinary excretion of 8 -isoprostane and a) apnoea-hypopnoea index (AHI; $r=0.55 ; p=0.0004)$, b) percentage of time with arterial oxygen saturation $\left(\mathrm{Sa}_{1} \mathrm{O}_{2}\right)<90 \%(r=0.40 ; p=0.02)$, c) serum levels of high-sensitivity $\mathrm{C}$-reactive protein ( $\left.h s C R P ; r=0.5 ; p=0.002\right)$, and d) body mass index (BMl; $r=0.4 ; p=0.02)$ in patients with obstructive sleep apnoea $(n=40)$.

higher than in lean or obese subjects without OSA, or patients with mild OSA. Overnight urinary excretion of 8-isoprostane was correlated with the severity of OSA, duration of hypoxia during sleep period, BMI and serum levels of hsCRP in patients with OSA. In addition, the severity of OSA was an independent predictor of overnight urinary excretion of 8isoprostane. Moreover, nCPAP significantly decreased both overnight urinary excretion of 8-isoprostane and serum levels of hsCRP in patients with moderate-to-severe OSA. Therefore, these findings are the first to show the significant association between systemic lipid peroxidation and inflammation in patients with OSA.

Isoprostanes are reliable biomarkers of lipid peroxidation and oxidative stress because of their stability and specificity. 8isoprostane is widely studied as a marker of oxidative stress in various respiratory diseases, including bronchial asthma, chronic obstructive pulmonary disease, cystic fibrosis and interstitial lung diseases [29-32]. Increased lipid peroxidation has also been reported in patients with OSA. CARPAGNANO and co-workers [24, 25] have reported increased production of 8isoprostane in the blood and exhaled breath condensates of patients with OSA. In addition, levels of other biomarkers of lipid peroxidation, oxidised low density lipoprotein cholesterol and thiobarbituric reactive substances are significantly higher in patients with OSA than in controls [33, 34]. Moreover, urinary 8-isoprostane is a risk marker for coronary artery disease [35]. In the present study, the overnight urinary excretion of 8-isoprostane was measured as a marker for oxidative stress during the sleep period in patients with OSA and it was found to be significantly elevated in these patients. These results suggest that lipid peroxidation is increased during sleep in patients with OSA.

Intermittent hypoxia observed in patients with OSA seems to resemble ischaemia-reperfusion. Several studies suggest that ischaemia-reperfusion represents oxidative stress and increases the generation of reactive oxygen species (ROS). On the basis of these findings, intermittent hypoxia is believed to increase the generation of ROS in patients with OSA [36]. In fact, production of superoxide by neutrophils and monocytes from patients with OSA is increased [19, 20, 33]. In the present study, the duration of hypoxia during sleep was found to be significantly correlated with overnight urinary excretion of 8isoprostane in patients with OSA. Moreover, changes in the duration of hypoxia during sleep after treatment with nCPAP were significantly correlated with changes in overnight urinary excretion of 8-isoprostane. Therefore, repeated apnoea-related hypoxia is an important factor in the overnight production of 8-isoprostane in patients with OSA. 

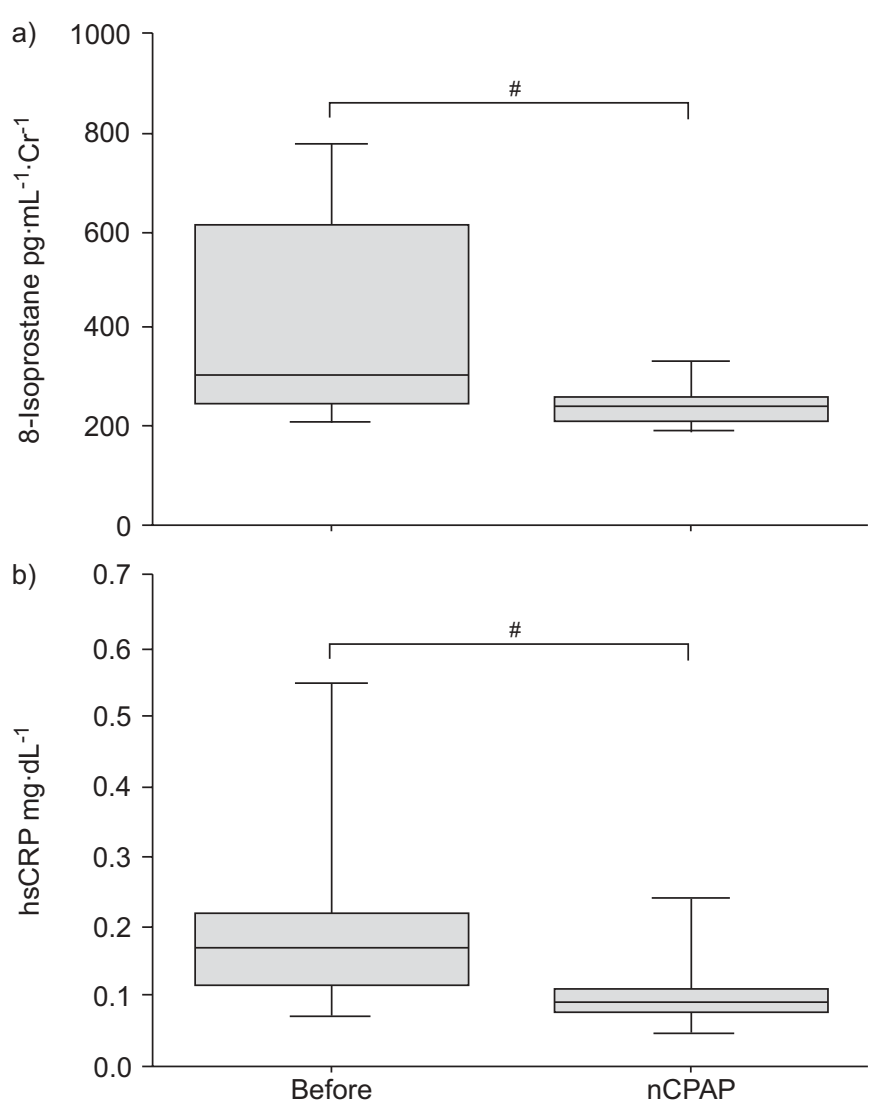

FIGURE 3. Effect of nasal continuous positive airway pressure (nCPAP) on a) overnight urinary excretion of 8-isoprostane and b) serum levels of high-sensitivity C-reactive protein (hsCRP) in patients with moderate-to-severe obstructive sleep apnoea (OSA; $n=20)$. Patients with moderate-to-severe OSA were treated with nCPAP for 3 months. The boundaries of each box represent the interquartile range (25th-75th centiles), the horizontal line inside the box represents the median, and the error bars outside the box represent the $95 \%$ confidence intervals of the mean. \#: $p<0.03$

Basic research studies have found that oxidative stress is critical for activation of nuclear factor (NF)- $\mathrm{B}$ [37]. Activation of NF- $\mathrm{KB}$ induces expression of pro-inflammatory cytokines, including IL-6, which stimulate the production of CRP by the liver [38]. Several clinical studies have suggested that oxidative stress markers are associated with CRP levels in healthy subjects without coronary artery disease [39-41]. In addition, a previous study has shown that oxidative stress is related to airway inflammation in patients with OSA because levels of exhaled 8-isoprostane were correlated with levels of IL-6 in exhaled breath condensates [24]. The present authors have confirmed and extended the close relationship between systemic oxidative stress and inflammation in patients with OSA by demonstrating that overnight urinary excretion of 8isoprostane was significantly correlated with serum levels of hsCRP.

Inflammation is associated with increased vascular production of ROS [42]. Specimens obtained at directional coronary atherectomy from patients with higher serum hsCRP levels showed greater immunoreactivity for CRP [42]. In addition, CRP directly enhances the expression of the reduced forms of nicotinamide adenine dinucleotide/nicotinamide adenine dinucleotide phosphate oxidase $\mathrm{p} 22^{\text {phox }}$ protein, as well as the generation of ROS in cultured human coronary artery smooth muscle cells [43]. Moreover, expression of 8-isoprostane is increased in atheroma [44]. These results suggest that CRP may directly promote oxidative stress.

Once increased systemic inflammation and oxidative stress are established, a vicious circle may develop in which oxidative stress can induce CRP production and increased CRP levels can in turn enhance oxidative stress. In addition, a recent study has suggested that visceral obesity is an important factor for enhanced oxidative stress in males [45]. Since repeated apnoearelated hypoxia and visceral obesity are present in patients with OSA, oxidative stress may promote both systemic lipid peroxidation and inflammation. Thus, the combination of visceral obesity and OSA may exacerbate oxidative stress and fuel a vicious circle between lipid peroxidation and inflammation.

Previous studies have shown that elevated levels of both systemic and airway oxidative stress are reduced by nCPAP therapy $[19,20,25]$. In the present study, it was also confirmed that nCPAP significantly decreases both overnight urinary excretion of 8-isoprostane and serum levels of hsCRP in patients with moderate-to-severe OSA. Moreover, changes in overnight urinary excretion of 8-isoprostane after treatment with nCPAP were correlated with changes in serum levels of hsCRP in these patients. Therefore, these results imply that nCPAP might be useful for decreasing systemic lipid peroxidation and inflammation in patients with OSA.

In the present study, no significant differences were found in the serum levels of hsCRP between lean subjects without OSA, obese subjects without OSA, and patients with mild OSA. The small number of subjects may explain the lack of significant differences between these groups. Moreover, the degree of adiposity in the presented subjects may not be sufficient to establish an independent effect of obesity on hsCRP levels. However, there was a significant difference in urinary levels of 8-isoprostane between lean subjects without OSA, obese subjects without OSA and patients with mild OSA. The excretion of higher levels of urinary 8-isoprostane in obese patients with mild OSA compared with obese subjects without OSA is consistent with both OSA and obesity impacting on oxidative stress and lipid peroxidation. In addition, AHI was an independent factor predicting the levels of urinary excretion of 8-isoprostane. Therefore, the current authors speculate that 8 -isoprostane may be a more sensitive marker for the presence of obesity and OSA than serum levels of hsCRP.

Limitations of the present study include the recruitment of only male subjects and the relatively small numbers of patients with OSA and control subjects. In addition, the effects of nCPAP on overnight urinary excretion of 8 -isoprostane and serum levels of hsCRP in patients with OSA were not examined using a randomised, placebo-controlled design. However, significant correlations were found between changes in AHI and changes in overnight urinary excretion of 8isoprostane and serum levels of hsCRP after treatment with nCPAP in patients with moderate-to-severe OSA. Therefore, nCPAP might decrease both overnight urinary excretion of 
8-isoprostane and serum levels of hsCRP in patients with moderate-to-severe OSA. The effects of nCPAP on these variables should be examined in a large, placebo-controlled study.

In conclusion, the present authors first demonstrated that overnight urinary excretion of 8-isoprostane and serum levels of high sensitivity C-reactive protein are elevated and correlated in patients with obstructive sleep apnoea. Moreover, obstructive sleep apnoea severity and obesity acted independently to elevate overnight urinary excretion of 8isoprostane. As systemic lipid peroxidation by oxidative stress and inflammation are increased, obstructive sleep apnoea may increase the risks of cardiovascular morbidity in obese individuals. In addition, nasal continuous positive airway pressure might be useful for decreasing these risks by, in part, reducing systemic lipid peroxidation and inflammation in patients with obstructive sleep apnoea.

\section{ACKNOWLEDGEMENTS}

The authors would like to thank $H$. Takeuchi for skilful technical assistance.

\section{REFERENCES}

1 Shahar E, Whitney CW, Redline S, et al. Sleep-disordered breathing and cardiovascular disease: cross-sectional results of the Sleep Heart Health Study. Am J Respir Crit Care Med 2001; 163: 19-25.

2 Peker Y, Hedner J, Norum J, Kraiczi H, Carlson J. Increased incidence of cardiovascular disease in middle-aged men with obstructive sleep apnea. A 7-year follow up. Am J Respir Crit Care Med 2002; 166: 159-165.

3 Marin JM, Carrizo SJ, Vicente E, Agusti AG. Long-term cardiovascular outcomes in men with obstructive sleep apnoea-hypopnoea with or without treatment with continuous positive airway pressure: an observational study. Lancet 2005; 365: 1046-1053.

4 Marti S, Sampol G, Munoz X, et al. Mortality in severe sleep apnoea/hypopnoea syndrome patients: impact of treatment. Eur Respir J 2002; 20: 1511-1518.

5 Libby P, Ridker PM, Maseri A. Inflammation and atherosclerosis. Circulation 2002; 105: 1135-1143.

6 Glass CK, Witztum JL. Atherosclerosis. The road ahead. Cell 2001; 104: 503-516.

7 Haverkate F, Thompson SG, Pyke SD, Gallimore JR, Pepys MB. Production of C-reactive protein and risk of coronary events in stable and unstable angina. European Concerted Action on Thrombosis and Disabilities Angina Pectoris Study Group. Lancet 1997; 349: 462-466.

8 Lindahl B, Toss H, Siegbahn A, Venge P, Wallentin L. Markers of myocardial damage and inflammation in relation to long-term mortality in unstable coronary artery disease. FRISC Study Group. Fragmin during instability in coronary artery disease. N Engl J Med 2000; 343: 1139-1147.

9 Burke AP, Tracy RP, Kolodgie F, et al. Elevated C-reactive protein values and atherosclerosis in sudden coronary death: association with different pathologies. Circulation 2002; 105: 2019-2023.

10 Yokoe T, Minoguchi K, Matsuo H, et al. Elevated levels of C-reactive protein and interleukin-6 in patients with obstructive sleep apnea syndrome are decreased by nasal continuous positive airway pressure. Circulation 2003; 107: 1129-1134.

11 Minoguchi K, Tazaki T, Yokoe T, et al. Elevated production of tumor necrosis factor-alpha by monocytes in patients with obstructive sleep apnea syndrome. Chest 2004; 126: 1473-1479.

12 Entzian P, Linnemann K, Schlaak M, Zabel P. Obstructive sleep apnea syndrome and circadian rhythms of hormones and cytokines. Am J Respir Crit Care Med 1996; 153: 1080-1086.

13 Chin K, Nakamura T, Shimizu K, et al. Effects of nasal continuous positive airway pressure on soluble cell adhesion molecules in patients with obstructive sleep apnea syndrome. Am J Med 2000; 109: 562-567.

14 Schulz R, Hummel C, Heinemann S, Seeger W, Grimminger F. Serum levels of vascular endothelial growth factor are elevated in patients with obstructive sleep apnea and severe nighttime hypoxia. Am J Respir Crit Care Med 2002; 165: 67-70.

15 Tazaki T, Minoguchi K, Yokoe T, et al. Increased levels and activity of matrix metalloproteinase-9 in obstructive sleep apnea syndrome. Am J Respir Crit Care Med 2004; 170: 1354-1359.

16 Minoguchi K, Yokoe T, Tazaki T, et al. Increased carotid intima-media thickness and serum inflammatory markers in obstructive sleep apnea. Am J Respir Crit Care Med 2005; 172: 625-630.

17 Dean RT, Wilcox I. Possible atherogenic effects of hypoxia during obstructive sleep apnea. Sleep 1993; 16: Suppl. 8, S15-S22.

18 Lavie L. Obstructive sleep apnoea syndrome - oxidative stress disorder. Sleep Med Rev 2003; 7: 35-51.

19 Schulz R, Mahmoudi S, Hattar K, et al. Enhanced release of superoxide from polymorphonuclear neutrophils in obstructive sleep apnea. Impact of continuous positive airway pressure therapy. Am J Respir Crit Care Med 2000; 162: 566-570.

20 Dyugovskaya L, Lavie P, Lavie L. Increased adhesion molecules expression and production of reactive oxygen species in leukocytes of sleep apnea patients. Am J Respir Crit Care Med 2002; 165: 934-939.

21 Harrison D, Griendling KK, Landmesser U, Hornig B, Drexler $\mathrm{H}$. Role of oxidative stress in atherosclerosis. Am J Cardiol 2003; 91: 7A-11A.

22 Dhalla NS, Temsah RM, Netticadan T. Role of oxidative stress in cardiovascular diseases. J Hypertens 2000; 18: 655-673.

23 Lawson JA, Rokach J, FitzGerald GA. Isoprostanes: formation, analysis and use as indices of lipid peroxidation in vivo. J Biol Chem 1999; 274: 24441-24444.

24 Carpagnano GE, Kharitonov SA, Resta O, et al. Increased 8isoprostane and interleukin- 6 in breath condensate of obstructive sleep apnea patients. Chest 2002; 122: 1162-1167.

25 Carpagnano GE, Kharitonov SA, Resta O, et al. 8Isoprostane, a marker of oxidative stress, is increased in exhaled breath condensate of patients with obstructive sleep apnea after night and is reduced by continuous positive airway pressure therapy. Chest 2003; 124: 1386-1392. 
26 The Report of an American Academy of Sleep Medicine Task Force. Sleep-related breathing disorders in adults: recommedations for syndrome definition and measurement techniques in clinical research. Sleep 1999; 22: 667-589.

27 Johns MW. A new method for measuring daytime sleepiness: the Epworth Sleepiness Scale. Sleep 1991; 14: 540-554.

28 Kazumi T, Kawaguchi A, Sakai K, Hirano T, Yoshino G. Young men with high-normal blood pressure have lower serum adiponectin, smaller LDL size, and higher elevated heart rate than those with optimal blood pressure. Diabetes Care 2002; 25: 971-976.

29 Montuschi P, Corradi M, Ciabattoni G, Nightingale J, Kharitonov SA, Barnes PJ. Increased 8-isoprostane, a marker of oxidative stress, in exhaled condensate of asthma patients. Am J Respir Crit Care Med 1999; 160: 216-220.

30 Montuschi P, Collins JV, Ciabattoni G, et al. Exhaled 8isoprostane as an in vivo biomarker of lung oxidative stress in patients with COPD and healthy smokers. Am J Respir Crit Care Med 2000; 162: 1175-1177.

31 Collins CE, Quaggiotto P, Wood L, O'Loughlin EV, Henry RL, Garg ML. Elevated plasma levels of F2 alpha isoprostane in cystic fibrosis. Lipids 1999; 34: 551-556.

32 Montuschi P, Ciabattoni G, Paredi P, et al. 8-Isoprostane as a biomarker of oxidative stress in interstitial lung diseases. Am J Respir Crit Care Med 1998; 158: 1524-1527.

33 Lavie L, Vishnevsky A, Lavie P. Evidence for lipid peroxidation in obstructive sleep apnea. Sleep 2004; 27: 123-128.

34 Barcelo A, Miralles C, Barbe F, Vila M, Pons S, Agusti AG. Abnormal lipid peroxidation in patients with sleep apnoea. Eur Respir J 2000; 16: 644-647.

35 Schwedhelm E, Bartling A, Lenzen H, et al. Urinary 8-isoprostaglandin F2alpha as a risk marker in patients with coronary heart disease: a matched case-control study. Circulation 2004; 109: 843-848.

36 Prabhakar NR. Sleep apneas: an oxidative stress? Am J Respir Crit Care Med 2002; 165: 859-860.

$37 \mathrm{Li} \mathrm{Q}$, Verma IM. NF-kappaB regulation in the immune system. Nat Rev Immunol 2002; 2: 725-734.

38 Hansson GK. Inflammation, atherosclerosis, and coronary artery disease. N Engl J Med 2005; 352: 1685-1695.

39 Yasunari K, Maeda K, Nakamura M, Yoshikawa J. Oxidative stress in leukocytes is a possible link between blood pressure, blood glucose, and C-reactive protein. Hypertension 2002; 39: 777-780.

40 Abramson JL, Hooper WC, Jones DP, et al. Association between novel oxidative stress markers and C-reactive protein among adults without clinical coronary heart disease. Atherosclerosis 2005; 178: 115-121.

41 Lee DH, Jacobs Jr DR. Association between serum gammaglutamyltransferase and C-reactive protein. Atherosclerosis 2005; 178: 327-330.

42 Madamanchi NR, Vendrov A, Runge MS. Oxidative stress and vascular disease. Arterioscler Thromb Vasc Biol 2005; 25: 29-38.

43 Kobayashi S, Inoue N, Ohashi $\mathrm{Y}$, et al. Interaction of oxidative stress and inflammatory response in coronary plaque instability: important role of C-reactive protein. Arterioscler Thromb Vasc Biol 2003; 23: 1398-1404.

44 Waddington EI, Puddey IB, Mori TA, Croft KD. Similarity in the distribution of $\mathrm{F}(2)$-isoprostanes in the lipid subfractions of atherosclerotic plaque and in vitro oxidised low density lipoprotein. Redox Rep 2002; 7: 179-184.

45 Urakawa H, Katsuki A, Sumida Y, et al. Oxidative stress is associated with adiposity and isulin resistance in men. $J$ Clin Endocrinol Metab 2003; 88: 4673-4676. 